\title{
Halftone to Continuous-Tone Conversion of Error-Diffusion Coded Images
}

\author{
Søren Hein, Member, IEEE, and Avideh Zakhor, Member, IEEE
}

\begin{abstract}
We consider the problem of reconstructing a continuous-tone (contone) image from its halftoned version, where the halftoning process is done by error diffusion. We present an iterative nonlinear decoding algorithm for halftoneto-contone conversion and show simulation results that compare the performance of the algorithm to that of conventional linear low-pass filtering. We find that the new technique results in subjectively superior reconstruction. As there is a natural relationship between error diffusion and $\Sigma \Delta$ modulation, our reconstruction algorithm can also be applied to the decoding problem for $\Sigma \Delta$ modulators.
\end{abstract}

\section{INTRODUCTION}

$\mathbf{H}$ ALFTONING is the process of converting continuoustone (contone) images into a form suitable for display on binary devices [1]-[4]. Halftoning is important because images such as photographs can have a large dynamic range, whereas display devices such as newspaper printers, laser printers, and some computer screens can only display binary images. The idea behind halftoning is to create a binary image whose lowfrequency content approximates that of a contone image and whose high-frequency content is not visibly correlated with the low-frequency content. At a suitable distance, the human visual system then acts approximately as a low-pass filter.

The halftoning process has been used in the printing industry for over a hundred years, and a large body of practical and theoretical knowledge has been accumulated [2]. The work reported in this paper and many others [5], [6] is mainly concerned with digital or electronic halftoning rather than manual halftoning, which involves contact screens. In the former problem type, an image is manipulated as a 2-D array of discrete samples, whereas in the latter problem type, parameters such as the sizes and shapes of ink dots can be varied. For simplicity, we only consider black-and-white (B/W) contone images.

A number of algorithms for halftoning images are available. The reader is referred to reviews such as [2]-[4] for a detailed discussion of the relative merits of these algorithms. In this paper, we consider the error diffusion (ED) algorithm [7] and address the problem of reconstructing a contone image from its halftoned version. This problem arises when high-

\footnotetext{
Manuscript received June 22, 1992; revised November 16, 1993. The associate editor coordinating the review of this paper and approving it for publication was Dr. Frederick Mintzer. This work was supported by NSF grant MIP-9057466, ONR young investigator award N00014-92-J-1732, Kodak, and Analog Devices.

S. Hein is with Siemens AG, Semiconductor Division, München, Germany.

A. Zakhor is with the Department of Electrical Engineering and Computer Sciences.University of California at Berkeley, Berkeley, CA 94720 USA

IEEE Log Number 9407605.
}

quality images are needed for immediate viewing or when the underlying contone image needs to be decimated, interpolated, extrapolated, linearly or nonlinearly filtered and enhanced, compressed [8], or manipulated in any other way. In these situations, it is natural to reconstruct the contone image, perform the desired operations on it, and halftone the result. Finally, in many applications such as facsimile and desk-top publishing, it may be desired to convert back and forth between different halftone images for display on printers and monitors with varying dynamic range and resolution characteristics.

There are several existing approaches to halftone-to-contone conversion. Fan [9] describes a logic-filtering approach for screened halftone images. In [8], Ting and Riskin present, among other results, a novel conversion approach based on error-diffused halftone images. Their approach is to decode each halftoned pixel based on the $3 \times 3$ neighborhood of the pixel; the decoded contone pixel value is obtained from a look-up table whose contents have previously been optimized through training on a suite of test images.

The most common approach to halftone-to-contone conversion is low-pass filtering [2]. However, there is more information in a halftone image than is extracted by a linear filter because linear filtering does not use knowledge of the detailed mechanism by which the halftone image was generated. Our goal is to use this extra information to obtain sharper and more pleasing contone reconstructions. This goal is achieved with an iterative, nonlinear algorithm based on projections onto convex sets (POCS) [10]. Our simulations show that the algorithm results in higher reconstruction quality than linear filtering. The theory of POCS has previously been applied to many signal and image reconstruction problems [11], including reconstruction of contone images from screened halftones [6], [9].

Our proposed algorithm is inspired by our work on nonlinear decoding for $\Sigma \Delta$ modulators [12], [13]. We thus exploit the relationship between ED and $\Sigma \Delta$ modulation first noted in [1].

The paper is organized as follows. Section II describes ED, and Section III describes our proposed reconstruction algorithm. Section IV shows sample results of our proposed algorithm, and Section V contains a summary.

\section{MODIFIED ERROR DIFFUSION}

In Section II-A, we give a brief, general introduction to error diffusion, and in Sections II-B and C, we lay the groundwork for the reconstruction algorithm described in Section III. In Section II-B, we discuss the choice of a computational procedure (CP) for ED halftoning. Although our chosen CP differs 


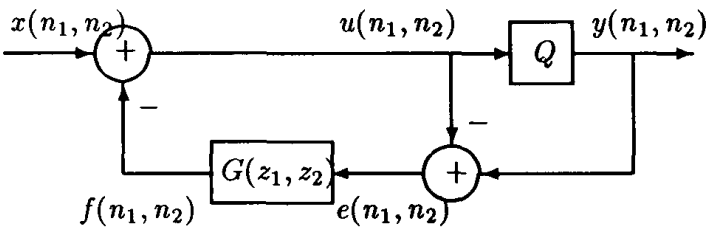

Fig. 1. Discrete-time model of an error diffusion encoder.

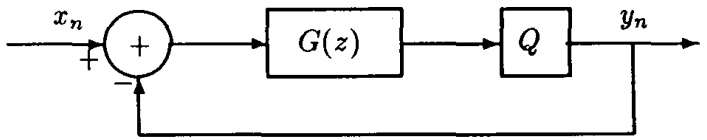

Fig. 2. Discrete-time model of the interpolative $\Sigma \Delta$ encoder.

slightly from the traditional one, it tremendously simplifies our reconstruction algorithm. In Section II-C, we derive the space-domain description of ED that results from our choice of $\mathrm{CP}$.

\section{A. Introduction}

Fig. 1 shows a diagram of an ED encoder that is a 2D discrete-time system. For comparison, Fig. 2 shows an interpolative $\Sigma \Delta$ modulator that is a $1-D$ system used in analog-to-digital conversion. Similar to a $\Sigma \Delta$ encoder, an ED encoder contains a 1-b quantizer $Q$ and a linear filter $G$ in a negative feedback loop and has a continuous-amplitude input and binary output. $Q$ is given by

$$
Q(u)=\left\{\begin{array}{cll}
0 & \text { for } & u \leq L_{\text {mid }} \\
L_{\text {high }} & \text { for } & u>L_{\text {mid }}
\end{array}\right.
$$

where $L_{\text {high }}=255$ and $L_{\text {mid }}=\frac{1}{2} L_{\text {high }}=127 \frac{1}{2}$, as we consider 8-b images. To be implementable, the 2-D filter $G\left(z_{1}, z_{2}\right)$ must have finite-extent input and output masks, and the system must be recursively computable [14]. For $G$, we will use the original Floyd-Steinberg input and output masks shown in Fig. 3 [7]. The equation for this ED filter is

$$
\begin{aligned}
f\left(n_{1}, n_{2}\right)= & e\left(n_{1}, n_{2}\right) \\
& +\frac{1}{16}\left[7 f\left(n_{1}-1, n_{2}\right)+f\left(n_{1}-1, n_{2}-1\right)\right. \\
& \left.+5 f\left(n_{1}, n_{2}-1\right)+3 f\left(n_{1}+1, n_{2}-1\right)\right] .
\end{aligned}
$$

The impulse response of the filter $G$ has the infinite-extent region of support (ROS) $R_{g}$ shown in Fig. 4(a). A number of improved filters with larger masks have been proposed to overcome problems with halftoning artifacts [4]. However, for simplicity, we use the original filter throughout this paper. We assume that the masks are known. If this is not the case, an interesting filter estimation problem arises. For an approach to a similar problem, see [6].

\section{B. Computational Procedure for Modified Error Diffusion}

A 2-D discrete-time system such as an ED encoder requires a computational procedure that describes how to compute the system output from the encoder input [14]. In this section, we define the particular CP that we will use, which is slightly modified from the standard one in order to significantly

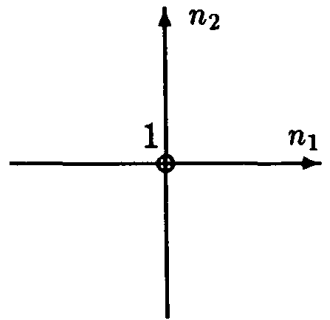

(a)

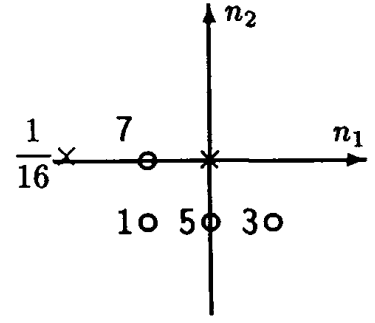

(b)
Fig. 3. (a) Input and (b) output masks for the filter $G$ in the Floyd-Steinberg ED encoder. The filter maps $\left\{e\left(n_{1}, n_{2}\right)\right\}$ into $\left\{f\left(n_{1}, n_{2}\right)\right\}$ in Fig. 1 .

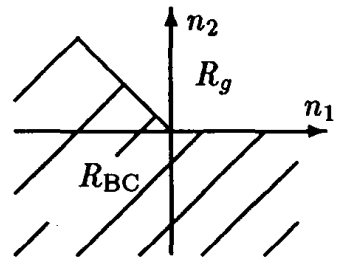

(a)

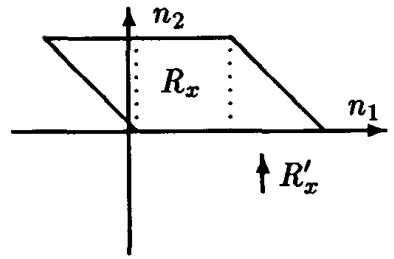

(b)
Fig. 4. (a) Region of support $R_{g}$ for the impulse response of $G\left(z_{1}, z_{2}\right)$; (b) region of support $R_{x}$ for the input, shown with dotted lines and wholly contained in the larger region $R_{x}^{\prime}$ over which the filter output must be calculated.

reduce the complexity of our reconstruction algorithm. The modification does not affect the quality of the halftoning. Our CP is inspired by the general procedure described in Lim [14] for linear systems and is outlined below. Using our procedure, we derive a space-domain description of the ED encoder relating the unknown encoder input $\left\{x\left(n_{1}, n_{2}\right)\right\}$, the known encoder output $\left\{y\left(n_{1}, n_{2}\right)\right\}$, and known spaceinvariant impulse responses determined by $G$.

To compute the output of the ED encoder for a given input, we must specify both boundary conditions and a recursive computational order [14]. We will begin with the boundary conditions that specify the region over which the encoder output is guaranteed to be zero, given that the encoder input is restricted to a specified region of support $R_{x}$ [14]. We will set $R_{x}=\left\{\left(n_{1}, n_{2}\right) \mid 0 \leq n_{1} \leq N-1,0 \leq n_{2} \leq N-1\right\}$, where $N$ is the image size. Let us pretend for a moment that the quantizer $Q$ in Fig. 1 is replaced by an open connection. As the filter input mask is a Kronecker $\delta$ function, the ROS of $\left\{f\left(n_{1}, n_{2}\right)\right\}$ is also $R_{g}$. Therefore, both the filter output $f\left(n_{1}, n_{2}\right)$ and the variable $u\left(n_{1}, n_{2}\right)$ are zero in the boundary condition region $R_{\mathrm{BC}}$ defined as the complement of $R_{g}$. Now, because $Q(0)=0$, reinserting $Q$ into the encoder over the region $R_{\mathrm{BC}}$ does not alter the encoder function over $R_{\mathrm{BC}}$ : $u\left(n_{1}, n_{2}\right)$ and, hence, $y\left(n_{1}, n_{2}\right)$ remain equal to zero over $R_{\mathrm{BC}}$. Therefore, the boundary condition region remains $R_{\mathrm{BC}}$ when $Q$ is inserted.

Having specified boundary conditions, we now complete our $\mathrm{CP}$ for the ED encoder by specifying a recursively computable order [14] in which encoder input samples can be processed. A natural order of computation through $R_{g}$ would be line by 
line. However, we only need the ED output over the region $R_{x}$ shown in Fig. 4(b). We can therefore discard any lines above $R_{x}$ in the $n_{2}$ direction as well as any samples with large enough values of $n_{1}$ so that they do not affect the encoder output over $R_{x}$. To calculate the encoder output over $R_{x}$, it thus suffices to go line by line through the finite region $R_{x}^{\prime}$ shown in Fig. 4(b). In contrast, the standard CP forces the quantization error to be zero outside of $R_{x}$, which leads to a violation of the governing difference equations over $R_{c}$; here, we have defined $R_{c}$ to be the part of $R_{x}^{\prime}$ that does not overlap with $R_{x}$.

Although our proposed CP simplifies the reconstruction, it has one potential problem: The decoder only has access to the encoder output over $R_{x}$, and due to the feedback, this encoder output may be affected by encoder outputs over $R_{c}$. However, we show in Appendix A that for our encoder, an encoder output sample is necessarily zero whenever the corresponding encoder input sample is zero. Therefore, the discarded encoder outputs over $R_{c}$ carry no information.

Although our proposed $\mathrm{CP}$ generates the same binary image over $R_{c}$ as the standard CP does, it generates a different image over $R_{x}$. This is because our procedure requires the encoder to process all of $R_{x}^{\prime}$, rather than only $R_{x}$, which affects the encoder states. Therefore, even though the encoder output is zero over $R_{c}$, the diffused errors are nonzero, and this affects the encoder outputs over the entire image. Nevertheless, we find that the two halftoning techniques result in subjectively similar quality.

\section{Resulting Space-Domain Description}

We now derive a space-domain description of the ED encoder. Referring to Fig. 1, we will write the quantizer input $u\left(n_{1}, n_{2}\right)$ as a linear combination of input samples $\left\{x\left(n_{1}, n_{2}\right)\right\}$ and output samples $\left\{y\left(n_{1}, n_{2}\right)\right\}$. For given filter input and output masks, we can use standard methods [14] to calculate the space-invariant impulse response $\left\{g_{x}\left(n_{1}, n_{2}\right)\right\}$ from the encoder input $\left\{x\left(n_{1}, n_{2}\right)\right\}$ to the quantizer input $\left\{u\left(n_{1}, n_{2}\right)\right\}$ and, similarly, the impulse response $\left\{g_{y}\left(n_{1}, n_{2}\right)\right\}$ from the encoder output $\left\{y\left(n_{1}, n_{2}\right)\right\}$ to the quantizer input $\left\{u\left(n_{1}, n_{2}\right)\right\}$. By definition of these impulse responses, we then have

$$
\begin{aligned}
u\left(n_{1}, n_{2}\right)= & \sum_{m_{1}} \sum_{m_{2}}\left[g_{x}\left(n_{1}-m_{1}, n_{2}-m_{2}\right) x\left(m_{1}, m_{2}\right)\right. \\
& \left.+g_{y}\left(n_{1}-m_{1}, n_{2}-m_{2}\right) y\left(m_{1}, m_{2}\right)\right]
\end{aligned}
$$

where the sums run from $-\infty$ to $+\infty$. Even though the quantizer $Q$ is nonlinear, the relationship (2) is linear. By inspection, $g_{x}(0,0)=1, g_{y}(0,0)=0$, and for $\left(n_{1}, n_{2}\right) \neq$ $(0,0), g_{x}\left(n_{1}, n_{2}\right)+g_{y}\left(n_{1}, n_{2}\right)=0 .{ }^{1}$ The summation limits in (2) can be reduced by making use of knowledge of the ROS's for the involved sequences. First, we have $x\left(m_{1}, m_{2}\right)=0$ for $\left(m_{1}, m_{2}\right) \notin R_{x}$. Second, we have the less obvious result that $y\left(m_{1}, m_{2}\right)=0$ for $\left(m_{1}, m_{2}\right) \notin R_{x}$, as shown in Appendix A; this is a form of stability result because it follows from the boundedness of the quantization error. Finally, the ROS's of

\footnotetext{
${ }^{1}$ When using the standard $\mathrm{CP}$, the quantizer input still depends linearly on encoder input and output.
}

the impulse responses can be calculated for given filter input and output masks. For instance, $g_{x}$ and $g_{y}$ for the masks shown in Fig. 3 are both zero whenever $n_{2}<0$ or $n_{1}+n_{2}<0$. Putting these facts together, we find that for our particular ED encoder

$$
\begin{aligned}
u\left(n_{1}, n_{2}\right)= & \sum_{m_{2}=0}^{M_{2}} \sum_{m_{1}=0}^{M_{1}}\left[g_{x}\left(n_{1}-m_{1}, n_{2}-m_{2}\right) x\left(m_{1}, m_{2}\right)\right. \\
& +g_{y}\left(n_{1}-m_{1}, n_{2}-m_{2}\right) y\left(m_{1}, m_{2}\right)
\end{aligned}
$$

where $M_{1}=\min \left\{n_{1}+n_{2}-m_{2}, N-1\right\}$ and $M_{2}=\min \left\{n_{2}\right.$, $N-1\}$.

\section{ReCONSTRUCTION ALgORITHM}

Our proposed algorithm for halftone-to-contone conversion is based on the theory of POCS [10]. We thus alternate between a space-domain projection $P_{1}$ onto a set $S_{1}$ and a frequencydomain projection $P_{2}$ onto a set $S_{2}$ to find an image invariant under both. The set $S_{1}$ contains all contone images that result in a specific, observed ED halftone image, and the set $S_{2}$ contains all band-limited contone images. The two sets are easily shown to be convex. ${ }^{2}$ To extract all information from an observed halftone image, we must find a signal $\hat{x} \in S_{1} \cap S_{2}$. According to the theory of POCS, such an element can be found from any initial guess $x_{0}$ by the iteration [10]

$$
\boldsymbol{x}_{n+1}=\left(P_{2} \circ P_{1}\right) \boldsymbol{x}_{n}, n \geq 0, \hat{\boldsymbol{x}}=P_{1} \hat{\boldsymbol{x}}=P_{2} \hat{\boldsymbol{x}}=\lim _{n \rightarrow \infty} \boldsymbol{x}_{n}
$$

In contrast, the traditional method of contone reconstruction is to apply a low-pass filter. This approach corresponds to enforcing the band limitation constraint and neglecting the space-domain constraint. In Sections III-A and B, we describe the two projections in more detail.

\section{A. Space-Domain Projection}

In this section, we present a matrix space-domain description of the error diffusion encoder based on (3). We then show that the projection $P_{1}$ of an image onto the image set $S_{1}$ is a quadratic programming (QP) problem.

We only have access to the encoder output image $\left\{y\left(n_{1}\right.\right.$, $\left.\left.n_{2}\right)\right\}$ and not the quantizer input sequence $\left\{u\left(n_{1}, n_{2}\right)\right\}$. Through (1) and (2), the halftone image thus provides us with a series of bounds on linear combinations of encoder input samples $\left\{x\left(n_{1}, n_{2}\right)\right\}$ of the form

$$
\begin{array}{cl}
u\left(n_{1}, n_{2}\right)>L_{\text {mid }} & \text { if } \quad y\left(n_{1}, n_{2}\right)=L_{\text {high }} \\
u\left(n_{1}, n_{2}\right) \leq L_{\text {mid }} & \text { if } \quad y\left(n_{1}, n_{2}\right)=0 .
\end{array}
$$$$
1 .
$$

${ }^{2} \mathrm{~A}$ set $S$ is convex if for all $\boldsymbol{a}, \boldsymbol{b} \in S, \alpha \boldsymbol{a}+(1-\alpha) \boldsymbol{b} \in S$ for any $0<\alpha<$ 
This equation can be written more succinctly in matrix notation. ${ }^{3}$ To this end, we introduce the $N^{2}$-dimensional vectors

$$
\begin{aligned}
\boldsymbol{x}= & \left\{x_{0}, \cdots, x_{N^{2}-1}\right\}^{\dagger} \\
= & \{x(0,0), \cdots, x(0, N-1) \vdots \\
& \cdots \vdots x(N-1,0), \cdots, x(N-1, N-1)\}^{\dagger} \\
\boldsymbol{y}= & \left\{y_{0}, \cdots, y_{N^{2}-1}\right\}^{\dagger} \\
= & \{y(0,0), \cdots, y(0, N-1) \vdots \\
& \cdots \vdots y(N-1,0), \cdots, y(N-1, N-1)\}^{\dagger}
\end{aligned}
$$

where $x^{\dagger}$ denotes the transpose of $x$. We also define the $N^{2} \times N^{2}$ block-Toeplitz lower-triangular matrix

$$
\boldsymbol{G}_{x}=\left[\begin{array}{ccccc}
\boldsymbol{G}_{0} & \mathbf{0} & \cdots & \mathbf{0} & \mathbf{0} \\
\boldsymbol{G}_{1} & \boldsymbol{G}_{0} & \cdots & \mathbf{0} & \mathbf{0} \\
\vdots & \vdots & & \vdots & \vdots \\
\boldsymbol{G}_{N-2} & \boldsymbol{G}_{N-3} & \cdots & \boldsymbol{G}_{0} & \mathbf{0} \\
\boldsymbol{G}_{N-1} & \boldsymbol{G}_{N-2} & \cdots & \boldsymbol{G}_{1} & \boldsymbol{G}_{0}
\end{array}\right]
$$

where 0 denotes the $N \times N$ zero matrix, and the $N \times$ $N$ Toeplitz matrix $\boldsymbol{G}_{n}$ is completely specified by its first row, $\left\{g_{x}(n, 0), g_{x}(n,-1) \cdots g_{x}(n,-N+1)\right\}$, and its first column, $\left\{g_{x}(n, 0), g_{x}(n, 1), \cdots, g_{x}(n, N-1)\right\}$. Finally, we define $I$ to be the $N^{2} \times N^{2}$ identity matrix and $Q$ to be the diagonal matrix whose $n$th diagonal element is -1 if $y_{n}=L_{\mathrm{high}}$ and +1 if $y_{n}=0$. We can then write (4) as

$$
\boldsymbol{Q} \boldsymbol{G}_{x} \boldsymbol{x} \leq \boldsymbol{Q}\left[L_{\mathrm{mid}} \boldsymbol{I}+\left(\boldsymbol{G}_{x}-\boldsymbol{I}\right) \boldsymbol{y}\right]
$$

where the inequality sign is to be taken coordinate wise. The space-domain projection problem can now be stated as follows: Given an arbitrary image vector $x$, find a vector $\hat{x}$ that satisfies (6) and minimizes the 2-norm distance $\|$ $\hat{\boldsymbol{x}}-\boldsymbol{x} \|_{2}^{2}$. This is a linearly constrained QP problem that can be solved with techniques available in the literature [15]. The QP problem is of the exact same form as the one stated in [12] for $\Sigma \Delta$ encoding. Therefore, the methods described in [12] for approximately solving the QP problem while drastically reducing computational complexity are directly applicable. Specifically, we can solve a number of QP subproblems of size $L_{\mathrm{QP}}$ rather than one QP problem of size $N^{2}$. We can also employ the idea described in [12] of overlapping QP subblocks in order to more closely approximate the solution to the actual $N^{2}$-dimensional QP problem.

\section{B. Frequency-Domain Projection}

The fundamental mathematical requirement on the frequency-domain projection $P_{2}$ is that it should be a 2norm projection. Furthermore, for good performance, the projection should be able to discriminate between images and "nonimages." Unfortunately, no such operator is presently

\footnotetext{
${ }^{3}$ With the conventional CP for error diffusion, the halftone image still induces bounds on linear combinations of encoder input samples. However. the structure of the bounds is simplified by our modification.
}

known. There is no inherent requirement that the desired operator be a frequency-domain based one [16]. However, we find a frequency-domain approach most attractive due to the approximate low-pass nature of many naturally occurring images [16].

We have considered using the discrete cosine transform (DCT) as the basis of a projection. Specifically, we can take the 2-D DCT of an image, set all but the low-frequency coefficients to zero, and take an inverse DCT. We have also considered a novel linear singular value decomposition (SVD)-based transform [17]. The SVD-based method for band limitation operates analogously to the DCT-based one and enjoys a certain maximal energy concentration property [17]. As we found that the SVD-based method performed better than the DCT-based one while requiring fewer coefficients [13], we discarded the DCT-based method.

We also considered linear filtering [18] and replaced the frequency-domain projection with the $9 \times 9$ Gaussian filter given by $h\left(n_{1}, n_{2}\right)=\exp \left[\left(n_{1}^{2}+n_{2}^{2}\right) /\left(2 \sigma^{2}\right)\right] / K$, where $-4 \leq n_{1}, n_{2} \leq+4, \sigma$ is the width, and $K$ is a normalization constant such that the tap values add up to one. As such linear filtering is not a projection, the theory of POCS does not guarantee convergence to an image invariant under both projections simultaneously, but subjectively good quality might nonetheless be achieved. Interestingly, the resulting iterative algorithm can actually be viewed as exactly solving an optimization problem associated with the space- and frequency-domain operators [19]. The idea is that the output of the composition of the two operators converges, even though the output of the individual operators does not.

\section{RESULTS}

In this section, we illustrate the performance of our reconstruction algorithm. We use the $512 \times 512$ Lenna image in Fig. 5 as our test case. For all POCS results, the QP block size for the space-domain projection is set to $L_{\mathrm{QP}}=$ 64 with an overlap of $50 \%$ between blocks. Whenever the frequency-domain projection is SVD-based, it is calculated as described in Apendix B. The number of POCS iterations is ten; a significantly smaller number of iterations results in worse performance. The Peak SNR (PSNR) between two $N \times N$ images $x$ and $\hat{x}$ is defined as

$$
\text { PSNR } \triangleq 10 \log _{10} \frac{255^{2}}{\frac{1}{N^{2}} \sum_{n_{1}=0}^{N-1} \sum_{n_{2}=0}^{N-1}\left[x\left(n_{1}, n_{2}\right)-\hat{x}\left(n_{1}, n_{2}\right)\right]^{2}} .
$$

Fig. 6 shows the effect of bandlimiting the Lenna image using the SVD-based projection. We find that the subjective quality of Fig. 6 is close to that of Fig. 5, although some detail is lost in the straws on the hat and in the hair falling down on the right. In addition, some ringing is introduced by the upper right edge of the hat brim.

Fig. 7 shows the halftoned version of Fig. 5 from which the reconstruction is done. We have also considered halftoning the SVD-limited image rather than the original image in order to ensure that at least one image is invariant under both the space- and frequency-domain projections so that the theory of 


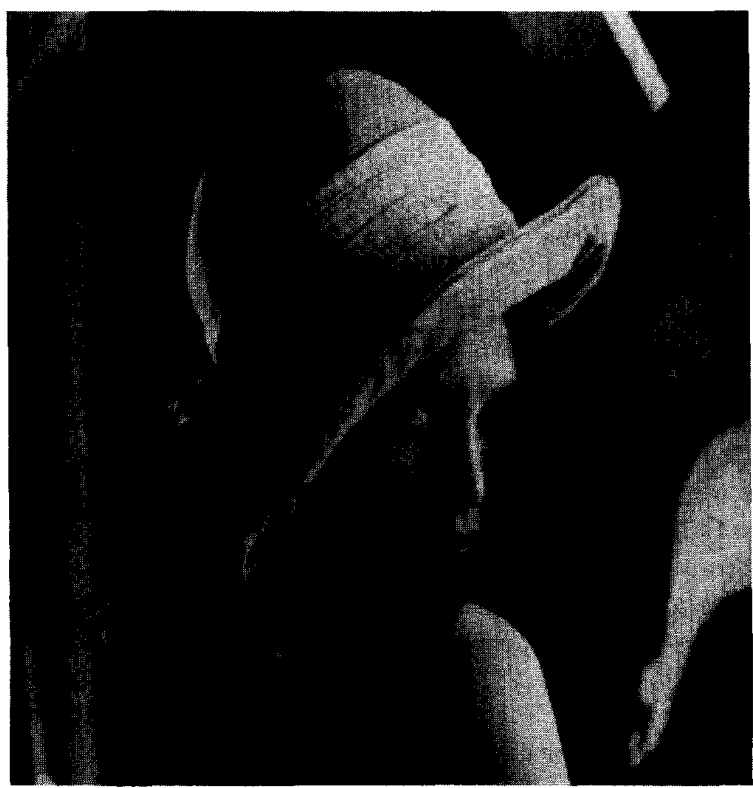

Fig. 5. Original $512 \times 5128$-b Lenna image.

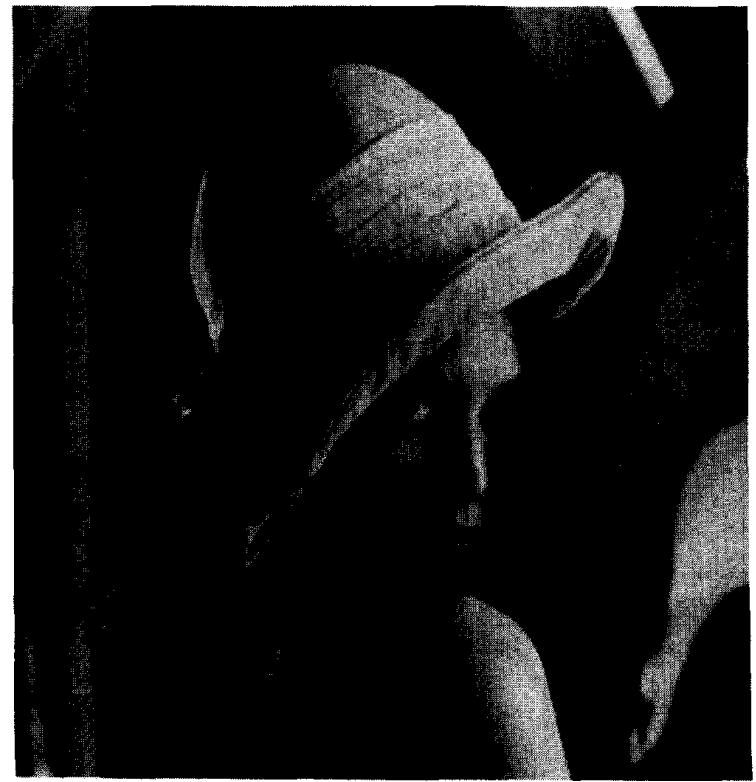

Fig. 6. SVD-limited Lenna image. The PSNR with respect to Fig. 5 is 32.3 dB.

POCS guarantees convergence. Such an approach would not be realistic in practice but would facilitate the investigation. We found that the results thus obtained were very close to the shown results.

Fig. 8 shows the effect of low-pass filtering the halftone image in Fig. 7 using a Gaussian $9 \times 9$ filter with $\sigma=2.0$. We find empirically that $\sigma=2.0$ results in the subjectively best low-pass filtering; choosing a larger $\sigma$ results in a more blurred image, whereas choosing a smaller $\sigma$ results in a more

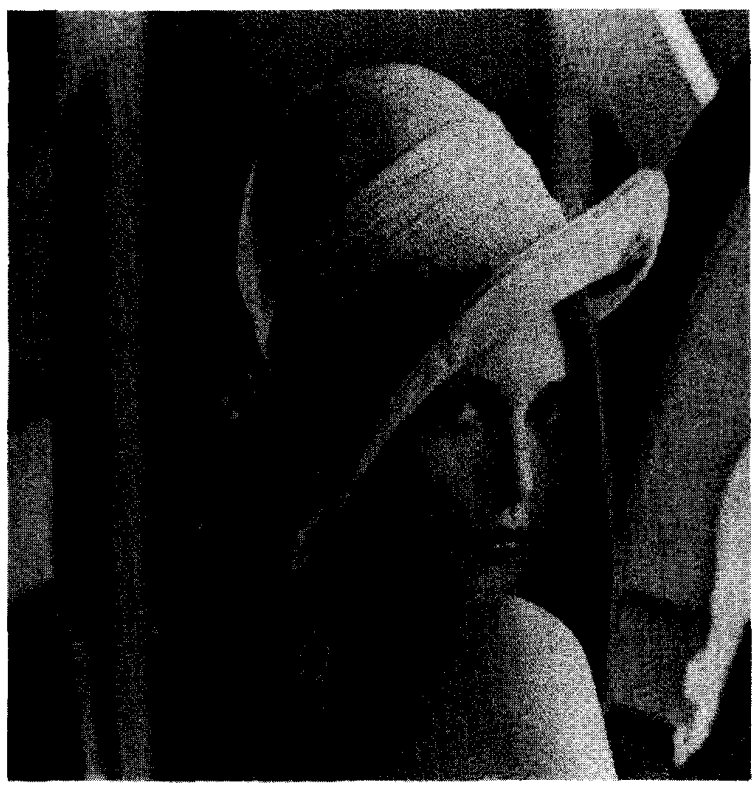

Fig. 7. Halftoned Lenna image.

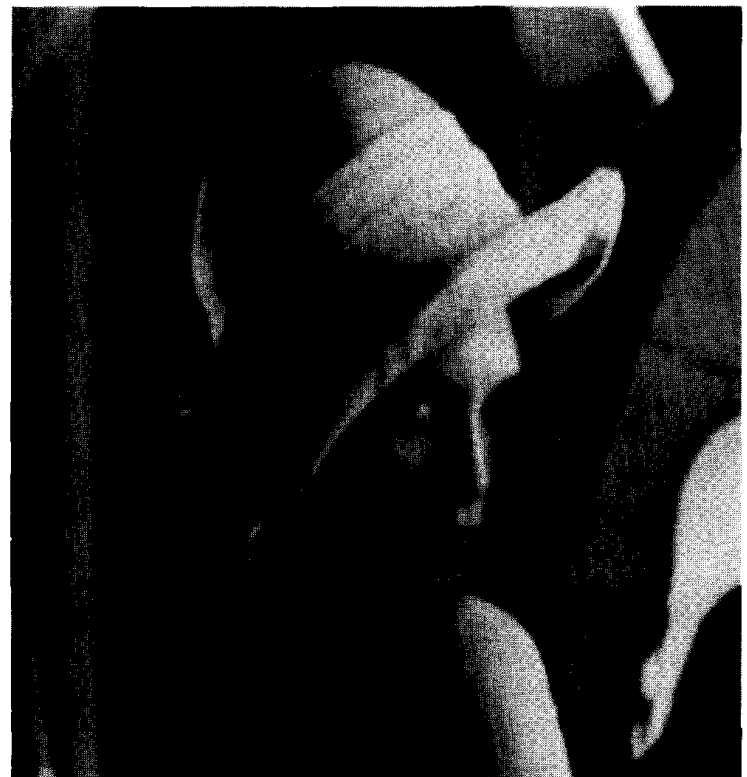

Fig. 8. Halftoned Lenna image filtered with a $9 \times 9$ Gaussian filter. The PSNR with respect to Fig. 5 is $27.4 \mathrm{~dB}$.

grainy appearance. Comparing Figs. 5 and 8, we see that linear filtering results in a visually pleasing but quite blurred image. Note for instance the blurring of the straws on the hat, the eyebrows, the eyes, and the upper brim of the hat. Note also the graininess of the left-most part of the cheek and the contouring in the background to the right of the cheek. The image in Fig. 8 is the benchmark against which we will compare the results of our reconstruction algorithm. 


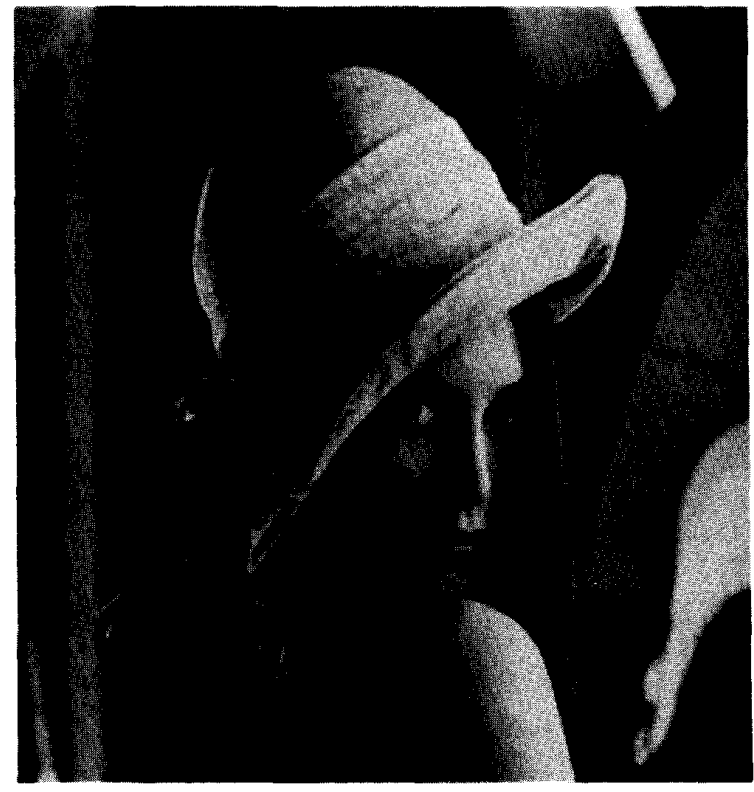

Fig. 9. Result of ten POCS iterations using the SVD as the band limitation. The PSNR with respect to Fig. 5 is $30.4 \mathrm{~dB}$.

Fig. 9 shows the result of applying the POCS algorithm to Fig. 7 using the SVD-based method as the band limitation. The reconstruction is quite sharp and visually pleasing compared with Fig. 8. In large magnification, however, Fig. 9 exhibits some visually objectionable artifacts in the form of a grainy texture in the lower right part of the face. In addition, ringing is present along the upper right edge of the hat brim as in Fig. 6 .

Figs. 10 and 11 show the results of applying the POCS algorithm with two different frequency-domain projections, both based on a $9 \times 9$ linear Gaussian filter whose $\sigma$ depends on the iteration number. The idea of varying $\sigma$ is inspired by [6]. By starting with a large $\sigma$, the initial iterations become quite low-pass and blurred, thus filtering out the halftone artifacts. Subsequently, decreasing $\sigma$ reduces the effect of the filter. In Fig. 10, we choose

$$
\sigma(n)= \begin{cases}2.0\left(1-\frac{n}{5}\right) & \text { for } n<5 \\ 0.4 & \text { for } n \geq 5\end{cases}
$$

where $n$ is the iteration number, and in Fig. 11, we choose

$$
\sigma(n)= \begin{cases}2.0\left(1-\frac{3 n}{20}\right) & \text { for } n<5 \\ 0.8 & \text { for } n \geq 5\end{cases}
$$

As expected, the image in Fig. 11 is somewhat more blurred than the one in Fig. 10 because $\sigma(n)$ in (8) is larger than $\sigma(n)$ in (7). On the other hand, Fig. 10 has a "pearly" appearance, with white dots appearing along the edges of the hat among other places. We ascribe this to the fact that as $\sigma$ decreases, the effect of the filter also decreases, and therefore, the space-domain projection dominates the algorithm. As the space-domain projection is not restricted to make smooth modifications to the iterates, the local white dots arise. The pearliness is absent in Fig. 11 due to the smaller bandwidth

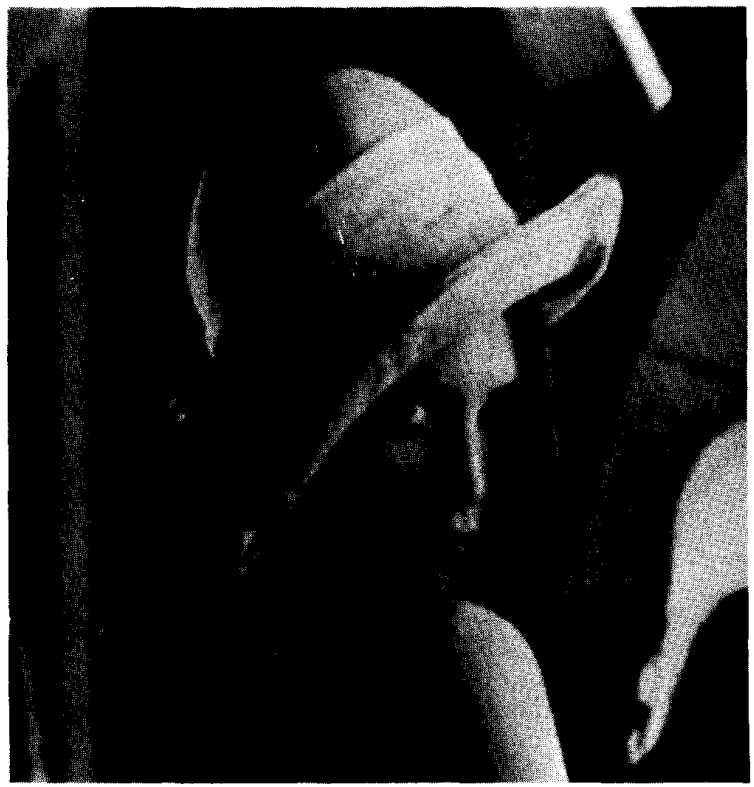

Fig. 10. Result of ten POCS iterations using linear filtering as the band limitation. The PSNR with respect to Fig. 5 is $29.4 \mathrm{~dB}$.

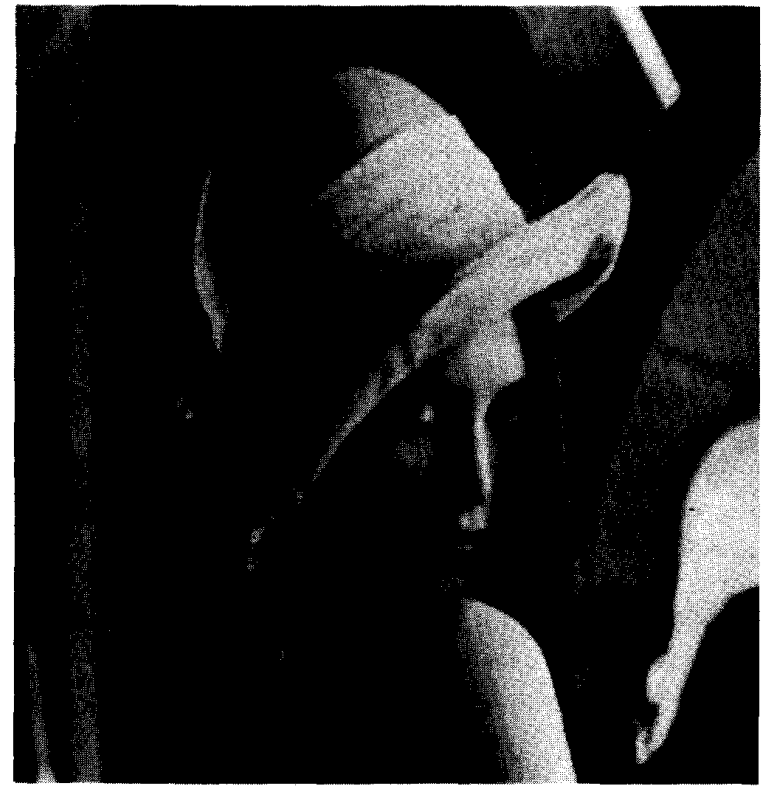

Fig. 11. Result of ten POCS iterations using a more narrow-band form of linear filtering as the band limitation. The PSNR with respect to Fig. 5 is $29.3 \mathrm{~dB}$.

of the filter employed. The choice of $\sigma(n)$ is thus a tradeoff between sharpness and pearliness. Both images are less sharp than Fig. 9. However, Figs. 10 and 11 are less grainy than Fig. 9 in large magnification.

It is interesting to compare Figs. 10 and 11 with Fig. 8, even though the bandwidths of the Gaussian filters used are not the same. Both Figs. 10 and 11 are sharper than Fig. 8 and do not 


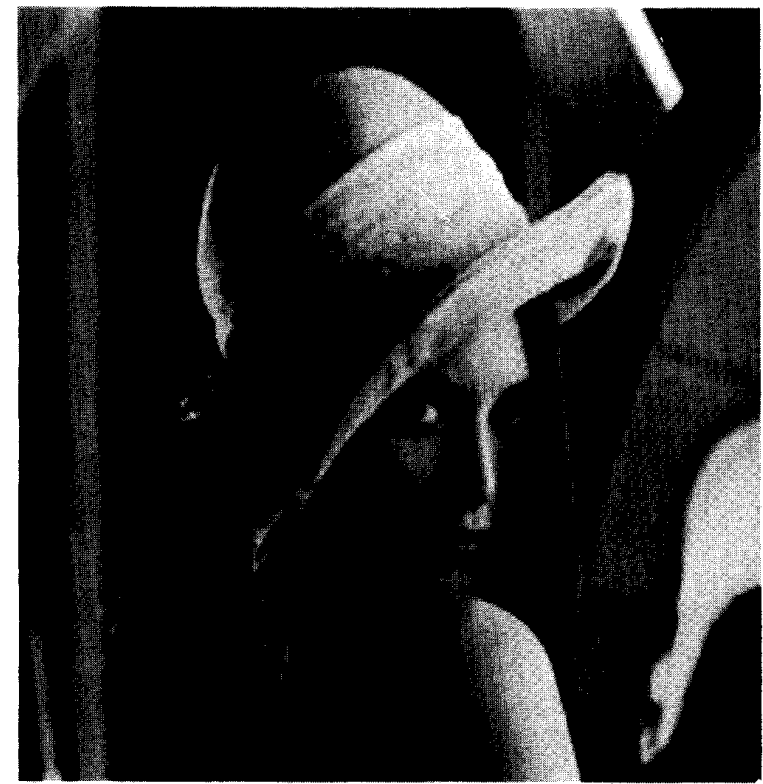

Fig. 12. Result of ten POCS iterations using both linear filtering and the SVD as the band limitation. The PSNR with respect to Fig. 5 is $29.3 \mathrm{~dB}$.

suffer from the graininess of Fig. 9. Using the POCS algorithm rather than linear filtering thus improves the reconstruction quality on both these counts. In addition, the filter used in the POCS iteration can be chosen to avoid pearly artifacts as discussed above.

Finally, Fig. 12 shows the result of applying the POCS algorithm with a mixed band limitation, namely, the linear filter characterized by (7), followed by the SVD-based method. The purpose of this is to combine the best of the SVD method and linear filtering for band limitation, namely, the convergence and sharpness properties of the SVD method and the smoothing effect of linear filtering. We find the image in Fig. 12 to be subjectively pleasing. The main artifacts of the image are in the white dots on the chin and the dark shade to the right of the nose. In addition, there is some graininess in the facial skin. On the other hand, Fig. 12 is sharper than Fig. 11. We find that although Fig. 12 is a good reconstruction, the best reconstructed image shown in this section is Fig. 8 .

To give some quantitative measure of the convergence rates involved in the above reconstructions, Fig. 13 shows the energy modification due to the frequency-domain projection as a function of iteration number for four variations on the frequency-domain projection, namely, the pure SVD method, linear filtering based on (7) and on (8), and the mixed band limitation. As expected, the pure SVD method has the best convergence curve. The other three frequency-domain operations all have about the same convergence performance. Neither of these curves correspond to converging algorithms, as linear filtering is not a projection. We emphasize that although it is mathematically desirable for an reconstruction algorithm to converge, an image processing algorithm should be judged primarily on its visual qualities and secondarily on strict convergence properties.

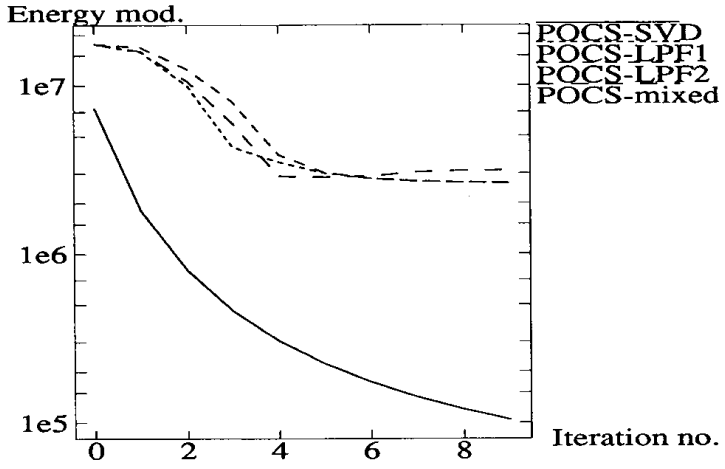

Fig. 13. Convergence curves for four band limitations as a function of POCS iteration number. Linear 1) and linear 2) curves refer to (7) and (8), respectively.

TABLE I

SUMmaRY OF IMAGE RECONSTRUCTION QUALITY

\begin{tabular}{|c||l|l|l|}
\hline Figure & Sharpness & Artifacts & Convergence \\
\hline 8 & low & graininess & N/A \\
9 & high & ringing & yes \\
10 & high & pearliness & no \\
11 & medium & low & no \\
12 & high & low & no \\
\hline
\end{tabular}

The subjective reconstruction quality of the presented images is tabulated in Table I. In summary, low-pass filtering can be used on its own as in Fig. 8, but this results in either graininess or blurriness, depending on the filter bandwidth. In contrast, Figs. 10 and 11 show the effect of linear filtering in a POCS iteration; the blurriness is clearly reduced, but pearliness results if the filter bandwidth is too large. Using the SVD method in a POCS iteration also reduces blurriness and results in fast convergence but introduces some ringing artifacts. Finally, in our opinion, a combination of the SVD method and linear filtering in a POCS iteration results in a reasonable compromise between sharpness and artifact-free images, as shown in Fig. 12.

\section{SUMMARY}

We have described a POCS-based iterative algorithm for reconstructing a contone image from its ED-encoded version. We showed simulation results that demonstrate that the proposed algorithm provides subjectively superior reconstruction to conventional linear filtering.

Alhtough this paper presents the mathematical framework for our algorithm and demonstrates its feasibility, it does not provide definitive answers to the contone reconstruction problem. Our conclusions are thus tentative and require psychovisual test for verification. In addition, the behavior of the algorithm with a number of different error diffusion filters 
could fruitfully be examined. Other questions include the optimal design of ED filters in the context of the presented algorithm and the problem of reconstruction when the ED filter is unknown. Reducing the computational complexity of the algorithm, particularly the space-domain projection, is also an interesting problem. The techniques presented in [20] may be relevant in this respect. Finally, the absence of a completely satisfactory frequency-domain projection is a problem whose solution would have implications not only for our algorithm but for other applications as well.

\section{APPENDIX A}

\section{STABILITY OF ERROR DIFFUSION ENCODERS}

We will show that the quantization error in an ED encoder is bounded, provided the following:

1) The input mask is a Kronecker $\delta$-function.

2) The absolute sum of the output mask coefficients is at most 1.

3) All input samples are in the range $\left[0, L_{\text {high }}\right]$.

Using the same technique, we then show that if an input sample $x\left(n_{1}, n_{2}\right)$ equals zero, the corresponding output sample $y\left(n_{1}, n_{2}\right)$ also equals zero.

We show the claims by induction. We first note that if $\left(n_{1}\right.$, $\left.n_{2}\right) \in R_{\mathrm{BC}}$, then $e\left(n_{1}, n_{2}\right)=0$, which belongs to the interval [ $\left.L_{\text {mid }}-L_{\text {high }}, L_{\text {mid }}\right]=\left[-L_{\text {mid }},+L_{\text {mid }}\right]$. We next establish the induction step. Consider the evaluation of a particular quantizer input sample $u\left(n_{1}, n_{2}\right)$. The largest possible value of $u\left(n_{1}, n_{2}\right)$ occurs if the corresponding input sample $x\left(n_{1}, n_{2}\right)$ and the contributions of the quantization errors through $G\left(z_{1}\right.$, $x_{2}$ ) are all as large as possible. Thus, abbreviating "output mask coefficient" by "o.m.c.," we have

$$
\begin{aligned}
u\left(n_{1}, n_{2}\right)= & x\left(n_{1}, n_{2}\right) \\
& +\sum_{m_{1}, m_{2}} \text { o.m.c. } \cdot e\left(m_{1}, m_{2}\right) \\
& \leq \max x\left(n_{1}, n_{2}\right) \\
& +\max _{m_{1}, m_{2}}\left|e\left(m_{1}, m_{2}\right)\right| \cdot \sum_{m_{1}, m_{2}} \mid \text { o.m.c. } \mid \\
= & L_{\text {high }}+L_{\text {mid }},
\end{aligned}
$$

where we have used the upper bound on $x\left(n_{1}, n_{2}\right)$, the upper and lower bounds on the quantization error, and the upper bound on the absolute sum of the output mask coefficients. Similarly, we find that

$$
\begin{aligned}
u\left(n_{1}, n_{2}\right)= & x\left(n_{1}, n_{2}\right) \\
& +\sum_{m_{1}, m_{2}} \text { o.m.c. } \cdot e\left(m_{1}, m_{2}\right) \\
& \geq \min x\left(n_{1}, n_{2}\right) \\
& -\max _{m_{1}, m_{2}}\left|e\left(m_{1}, m_{2}\right)\right| \cdot \sum_{m_{1}, m_{2}} \mid \text { o.m.c. } \mid \\
= & -L_{\text {mid }} .
\end{aligned}
$$

Combining (9) and (10), we get that $u\left(n_{1}, n_{2}\right) \in\left[-L_{\text {mid }}\right.$, $\left.L_{\text {high }}+L_{\text {mid }}\right]$. Now, for all quantizer inputs in this range, we have that $e\left(n_{1}, n_{2}\right)=Q(u)-u \in\left[-L_{\text {mid }},+L_{\text {mid }}\right]$, which establishes the induction step and shows that the absolute error $\left|e\left(n_{1}, n_{2}\right)\right|$ is no larger than $L_{\text {mid }}$ under the stated assumptions.

Consider next the case of a zero input sample, $x\left(n_{1}, n_{2}\right)=$ 0 , as occurs for any pair $\left(n_{1}, n_{2}\right) \notin R_{x}$. The upper bound (9) on $u\left(n_{1}, n_{2}\right)$ can then be strengthened to $u\left(n_{1}, n_{2}\right) \leq L_{\text {mid }}$. As the quantizer output is zero for any quantizer input less than or equal to $L_{\text {mid }}$, the bound on $u\left(n_{1}, n_{2}\right)$ establishes that $y\left(n_{1}, n_{2}\right)=0$. In the absence of this result, the halftone outputs $y\left(n_{1}, n_{2}\right)$ outside of $R_{x}$ would carry information necessary to derive bounds on the input image from (3), and thus, it would not be possible to perform the time-domain projection using only $\left\{y\left(n_{1}, n_{2}\right)\right\}$ over $R_{x}$.

\section{APPENDIX B}

\section{TWO-DIMENSIONAL SVD BAND LIMITATION}

In this appendix, we describe the generalization to two dimensions of the SVD-based band limitation method presented in [17] for 1-D signals. As in [17], we denote the 1-D truncated discrete prolate spheroidal sequences (DPSS's) corresponding to a particular bandwidth and sample size $N$ by $\left\{\mathbf{u}_{\mathbf{0}}, \cdots, \mathbf{u}_{\mathbf{N}-1}\right\}$. The SVD transform $\left\{X\left(m_{1}, m_{2}\right)\right\}$ of an image $\left\{x\left(n_{1}, n_{2}\right)\right\}$ is then given by

$$
X\left(m_{1}, m_{2}\right)=\sum_{n_{1}=0}^{N-1} \sum_{n_{2}=0}^{N-1} x\left(n_{1}, n_{2}\right) u_{m_{1}}\left(n_{1}\right) u_{m_{2}}\left(n_{2}\right)
$$

with the inversion formula

$$
x\left(n_{1}, n_{2}\right)=\sum_{m_{1}=0}^{N-1} \sum_{n_{2}=0}^{N-1} X\left(m_{1}, m_{2}\right) u_{m_{1}}\left(n_{1}\right) u_{m_{2}}\left(n_{2}\right) .
$$

The image $\left\{x\left(n_{1}, n_{2}\right)\right\}$ can be bandlimited by restricting the summation limits in (12). In the special case of a separable band limitation, that is, if the summation limits on $m_{1}$ and $m_{2}$ are independent, the image $\left\{x\left(n_{1}, n_{2}\right)\right\}$ can be bandlimited successively in the two dimensions as follows:

$$
\begin{aligned}
x^{(1)}\left(n_{1}, n_{2}\right) & =\sum_{i=0}^{r-1}\left(\sum_{m_{1}=0}^{N-1} x\left(m_{1}, n_{2}\right) u_{i}\left(m_{1}\right)\right) u_{i}\left(n_{1}\right) \\
x^{(2)}\left(n_{1}, n_{2}\right) & =\sum_{j=0}^{r-1}\left(\sum_{m_{2}=0}^{N-1} x^{(1)}\left(n_{1}, m_{2}\right) u_{j}\left(m_{2}\right)\right) u_{j}\left(n_{2}\right) .
\end{aligned}
$$

This is the form in which we will use the SVD band limitation. The method is characterized by the bandwidth, the sample size $N$ defining the DPSS's, and the number of vectors $r$. Unlike most 1-D signals, there is no natural bandwidth for a given image; therefore, choosing the bandwidth must be done empirically. We find that a bandwidth oversampling factor [17] of 2.5 and a number $r=210$ of singular vectors gives adequate results for an image size of $N \times N=512 \times 512$.

\section{ACKNOWLEDGMENT}

The authors would like to thank Prof. E. Riskin for suggesting the problem and useful discussions. 


\section{REFERENCES}

[1] D. Anastassiou, "Error diffusion coding for A/D conversion," IEEE Trans. Circuits Syst., vol. 36, pp. 1175-1186, Sept. 1989.

[2] Y.-M. Chao, "An investigation into the coding of halftone pictures," Ph.D. thesis, Mass. Inst. of Technol., May 1982

[3] J. C. Stoffel and J. F. Moreland, "A survey of electronic techniques for pictorial image reproduction," IEEE Trans. Commun., vol. COM-29, pp. $1898-1925$, Dec. 1981

[4] R. A. Ulichney, Digital Halftoning. Cambridge, MA: MIT Press, 1987.

[5] A. Zakhor, "Reconstruction of multidimensional signals from multiple level threshold crossings," Ph.D. thesis, Mass. Inst. of Technology, Oct. 1987.

[6] M. Analoui and J. Allebach, "New results on reconstruction of continuous-tone from halftone," in Proc. Int. Conf. Acoustics, Speech Signal Processing, Mar. 1992, pp. 313-316.

[7] R. W. Floyd and L. Steinberg, "An adaptive algorithm for spatial greyscale," in SID Int. Symp. Dig. Tech. Papers, 1975, pp. 36-37.

[8] M. Y. Ting and E. A. Riskin, "Error diffused image compression using a halftone-to-grayscale decoder and predictive pruned tree-structured vector quantization." to be published in IEEE Trans. Image Processing, 1992.

[9] Z. Fan, "Retrieval of images from digital halftones," in Proc. Int. Symp. Circuits Syst., May 1992, pp. 2477-2480.

[10] D. C. Youla and H. Webb, "Image restoration by the method of convex projections: Part I-Theory," IEEE Trans. Med. Imaging, vol. MI-1, pp. 81-94, Oct. 1982.

[11] M. I. Sezan and H. Stark, "Image restoration by the method of convex projections: Part II-Applications and numerical results," IEEE Trans. Med. Imaging, vol. MI-1, pp. 95-101, Oct. 1982

[12] S. Hein and A. Zakhor, "Reconstruction of oversampled band-limited signals from Sigma Delta encoded binary sequences," IEEE Trans. Signal Processing, vol. 42, no. 4, pp. 899-911, Apr. 1994.

[13] Sigma Delta Modulators: Nonlinear Decoding Algorithms and Stability Analysis. Boston: Kluwer, 1993.

[14] J. S. Lim, Two-Dimensional Signal and Image Processing. Englewood Cliffs, NJ: Prentice-Hall, 1990.

[15] D. G. Luenberger, Linear and Nonlinear Programming. Reading, MA: Addison-Wesley, 2nd ed., 1984.

[16] P. L. Combettes and H. J. Trussell, "The use of noise properties in set theoretic estimation," IEEE Trans. Signal Processing, vol. 39, pp. 1630-1641, July 1991.

[17] S. Hein and A. Zakhor, "Theoretical and numerical aspects of an SVDbased method for band-limiting finite extent sequences," IEEE Trans. Signal Processing, vol. 42, no. 5, pp. 1227-1230, May 1994.

[18] R. Rosenholtz and A. Zakhor, "Iterative procedures for reduction of blocking effects in transform image coding," IEEE Trans. Circuits Syst. Video Technol., vol. 2, pp. 91-95, Mar. 1992.

[19] S. J. Reeves and S. L. Eddins, "Comments on "Iterative procedures for reduction of blocking effects in transform image coding," to be published inlEEE Trans. Circuits Systems Video Technol., 1993.
[20] S. Hein, "A fast block-based nonlinear decoding algorithm for Sigma Delta modulators," to be published in IEEE Trans. Signal Processing.

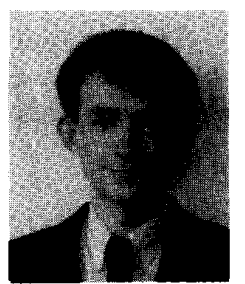

Saren Hein (M'93) was born in May 1968 in Copenhagen, Denmark. He received the M.S.c. degree in electrical engineering from the Technical University of Denmark in January 1989 and the $\mathrm{Ph} . \mathrm{D}$. degree in electrical engineering from the University of California at Berkeley in June 1992.

Since October 1992, he has worked for Siemens Corporate Research and Development, München, Germany. His current research interests include algorithmic aspects of oversampled A/D conversion and halftoning, signal reconstruction, band-limited extrapolation, and signal and image processing for medical, radar, and other applications. He has also worked on error-correction coding for satellite applications. He
communication.

While at Berkeley, Dr. Hein received the Eliahu Jury Award for outstanding research in the area of systems, communications, control, or signal processing and the Outstanding Graduate Student Instructor Award, both in 1992

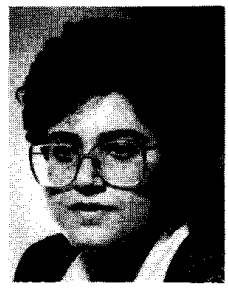

Avideh Zakhor (M'87) received the B.S. degree from California Institute of Technology, Pasadena, and the S.M. and Ph.D. degrees from the Massachusetts Institute of Technology, Cambridge, all in electrical engineering, in 1983, 1985, and 1987, respectively.

In 1988, she joined the Faculty at University of California at Berkeley, where she is currently Assistant Professor in the Department of Electrical Engineering and Computer Sciences. Her research interests are in the general area of signal processing and its applications to images and video, and biomedical data. She has been a consultant to a number of industrial organizations and holds four U.S. patents.

Ms. Zakhor was a General Motors scholar from 1982 to 1983 , received the Henry Ford Engineering Award and Caltech Prize in 1983, was a Hertz fellow from 1984 to 1988, received the Presidential Young Investigators (PYI) award, IBM junior faculty development award, and Analog Devices junior faculty development award in 1990, and the Office of Naval Research (ONR) young investigator award in 1992. She is currently an Associate Editor for IEEE Transactions on Image Processing and a member of the Technical Committee for Multidimensional Digital Signal Processing. 